Volume 136, Number 1, January 2008, Pages 133-140

S 0002-9939(07)09142-3

Article electronically published on October 12, 2007

\title{
NOTE ON A REMARKABLE SUPERPOSITION FOR A NONLINEAR EQUATION
}

\author{
PETER LINDQVIST AND JUAN J. MANFREDI
}

(Communicated by Juha M. Heinonen)

\begin{abstract}
We give a simple proof of - and extend - a superposition principle for the equation $\operatorname{div}\left(|\nabla u|^{p-2} \nabla u\right) \leq 0$, discovered by Crandall and Zhang. An integral representation comes as a byproduct. It follows that a class of Riesz potentials is $p$-superharmonic.
\end{abstract}

\section{INTRODUCTION}

The Newtonian potentials

$$
V(x)=c_{n} \int \frac{\rho(y) d y}{|x-y|^{n-2}}, \quad \rho \geq 0,
$$

are important examples of superharmonic functions in the $n$-dimensional Euclidean space, $n \geq 3$. They are obtained through a superposition of fundamental solutions

$$
\frac{A_{j}}{\left|x-y_{j}\right|^{n-2}}, \quad A_{j} \geq 0
$$

of the Laplace equation. The equation $\Delta V(x)=-\rho(x)$ holds.

For the $p$-Laplace equation

$$
-\operatorname{div}\left(|\nabla u|^{p-2} \nabla u\right)=0
$$

it was recently discovered by M. Crandall and J. Zhang that a similar superposition of fundamental solutions is possible. Indeed, they proved in [CZ] that sums like

$$
\sum A_{j}\left|x-a_{j}\right|^{\frac{p-n}{p-1}} \quad(2<p<n)
$$

are $p$-superharmonic functions, where $A_{j} \geq 0$. They also included exponents other than the natural $(p-n) /(p-1)$ and allowed $p$ to vary between 1 and $\infty$. The Riesz potentials

$$
\int \frac{\rho(y) d y}{|x-y|^{(n-p) /(p-1)}}
$$

appear as the limit of such sums.

Received by the editors September 18, 2006.

2000 Mathematics Subject Classification. Primary 35J60, 31C45.

This paper was written while the first author was visiting the University of Pittsburgh. He wishes to acknowledge the hospitality and the stimulating working atmosphere at the Department of Mathematics. The second author was partially supported by NSF award DMS-0500983. 
The purpose of our note is to give an alternative proof of the following theorem for the Riesz potentials

$$
V_{\alpha}(x)=\int|x-y|^{\alpha} \rho(y) d y .
$$

Theorem. Let $\rho \in C_{0}\left(\mathbb{R}^{n}\right), n \geq 2$, be a nonnegative function. We have three cases depending on $p$ :

(i) $\mathbf{2}<\mathbf{p}<\mathbf{n}$. The function $V_{\alpha}$ is p-superharmonic, if

$$
\frac{p-n}{p-1} \leq \alpha<0
$$

(ii) $\mathbf{p}>\mathbf{n}$. The function $V_{\alpha}$ is p-subharmonic, if

$$
\alpha \geq \frac{p-n}{p-1} .
$$

If $p=\infty$, we may take $\alpha \geq 1$.

(iii) $\mathbf{p}=\mathbf{n}$. The function

$$
V_{0}(x)=\int \log (|x-y|) \rho(y) d y
$$

is n-subharmonic.

Before proceeding, we make a comment about the case $1<p<2$, which exhibits a puzzling behaviour. While the fundamental solution

$$
|x-a|^{\frac{p-n}{p-1}}
$$

is $p$-superharmonic in the whole $\mathbb{R}^{n}$, the sum

$$
|x-a|^{\frac{p-n}{p-1}}+|x-b|^{\frac{p-n}{p-1}}
$$

is not, assuming of course that $a \neq b$. The sum is $p$-subharmonic when $x \neq a$ and $x \neq b$, but it is not $p$-subharmonic in the whole $\mathbb{R}^{n}$. A $p$-subharmonic function cannot take the value $+\infty$ in its domain of definition because of the comparison principle. This was about $p<2$.

We recall from $[\mathrm{L}]$ that $p$-superharmonic functions are defined as lower semicontinuous functions $v: \mathbb{R}^{n} \longrightarrow(0, \infty]$ that obey the comparison principle with respect to the $p$-harmonic functions. A more direct characterization is available for smooth functions. When $p \geq 2$ the function $v \in C^{2}\left(\mathbb{R}^{n}\right)$ is $p$-superharmonic if and only if

$$
-\operatorname{div}\left(|\nabla v(x)|^{p-2} \nabla v(x)\right) \geq 0
$$

at each point $x$. From the identity

$$
\operatorname{div}\left(|\nabla v|^{p-2} \nabla v\right)=|\nabla v|^{p-4}\left\{|\nabla v|^{2} \Delta v+(p-2) \Delta_{\infty} v\right\},
$$

where

$$
\Delta_{\infty} v=\sum_{i, j=1}^{n} \frac{\partial v}{\partial x_{i}} \frac{\partial v}{\partial x_{j}} \frac{\partial^{2} v}{\partial x_{i} \partial x_{j}}
$$

is the $\infty$-Laplacian operator, we can read off that the pointwise inequality

$$
|\nabla v|^{2} \Delta v+(p-2) \Delta_{\infty} v \leq 0
$$

is an equivalent characterization of $p$-superharmonic functions $v$ in $C^{2}\left(\mathbb{R}^{n}\right)$. (Incidentally, this is also valid in the case $1<p<2$. See [JLM].)

Thus we have a practical definition for functions of class $C^{2}$. The polar set $\Xi=\{x: v(x)=+\infty\}$ can be exempted if $v$ is lower semicontinuous in $\mathbb{R}^{n}$ and 
$v \in C^{2}\left(\mathbb{R}^{n} \backslash \Xi\right)$. An example is the fundamental solution $|x-a|^{(p-n) /(p-1)}, 1<p<$ $n$, where the point $x=a$ is exempted.

Finally, we mention that in Section 3 we need the concept of weak p-supersolution. We say that $v \in W_{\text {loc }}^{1, p}\left(\mathbb{R}^{n}\right)$ is a weak $p$-supersolution if

$$
\int_{\mathbb{R}^{n}}\left\langle|\nabla v|^{p-2} \nabla v, \nabla \varphi\right\rangle d x \geq 0
$$

holds for all nonnegative $\varphi \in C_{0}^{\infty}\left(\mathbb{R}^{n}\right)$. In [L] it was established that locally bounded $p$-superharmonic functions are weak $p$-supersolutions. On the other hand, lower semicontinuous weak $p$-supersolutions are $p$-superharmonic functions.

We thank the anonymous referee for a careful reading of the manuscript and for several suggestions that have improved its readability.

\section{Proof of the Theorem}

We assume $n \geq 2$. The following calculations are formal, but are easy to justify because $\alpha>2-n$. Notice that we have

$$
\alpha \geq \frac{p-n}{p-1}>2-n \text { when } p>2
$$

Differentiating

$$
V(x)=\int|x-y|^{\alpha} \rho(y) d y
$$

under the integral sign we obtain

$$
\frac{\partial V}{\partial x_{i}}=\alpha \int|x-y|^{\alpha-2}\left(x_{i}-y_{i}\right) \rho(y) d y
$$

and

$$
\begin{gathered}
\frac{\partial^{2} V}{\partial x_{i} \partial x_{j}}=\alpha(\alpha-2) \int|x-y|^{\alpha-4}\left(x_{i}-y_{i}\right)\left(x_{j}-y_{j}\right) \rho(y) d y \\
+\alpha \delta_{i j} \int|x-y|^{\alpha-2} \rho(y) d y .
\end{gathered}
$$

Aiming at $\Delta_{\infty} V$, we write the product of the integrals in

$$
\frac{\partial V}{\partial x_{i}} \frac{\partial V}{\partial x_{j}} \frac{\partial^{2} V}{\partial x_{i} \partial x_{j}}
$$

as a triple integral in disjoint variables. ${ }^{*}$ This yields the formula

$$
\begin{gathered}
\Delta_{\infty} V(x)=\alpha^{3} \int|x-c|^{\alpha-2} \rho(c) d c\left|\int\right| x-\left.\left.a\right|^{\alpha-2}(x-a) \rho(a) d a\right|^{2} \\
+\alpha^{3}(\alpha-2) \int|x-c|^{\alpha-2} \rho(c)\left\langle\frac{x-c}{|x-c|}, \int|x-a|^{\alpha-2}(x-a) \rho(a) d a\right\rangle^{2} d c
\end{gathered}
$$

in vector notation. Keeping $\alpha$ within the prescribed range we have only harmless singularities.

${ }^{*}$ The principle is clear from the example

$$
\left(\int e^{x} d x\right)^{2} \int e^{2 x} d x=\iiint e^{a+b+2 c} d a d b d c .
$$


By the Cauchy-Schwarz inequality we have

$$
\left|\left\langle\frac{x-c}{|x-c|}, \int|x-a|^{\alpha-2}(x-a) \rho(a) d a\right\rangle\right| \leq\left|\int\right| x-\left.a\right|^{\alpha-2}(x-a) \rho(a) d a \mid .
$$

In easily understandable notation we can therefore write the above formula as

$$
\Delta_{\infty} V(x)=\alpha^{3} A(x)+\alpha^{3}(\alpha-2) B(x)
$$

where

$$
0 \leq B(x) \leq A(x) .
$$

From this we can read off that $\Delta_{\infty} V(x) \geq 0$ when $\alpha \geq 1$. This settles the case $p=\infty$. There is a more succinct representation. Lagrange's identity

$$
|X \wedge Y|^{2}=\frac{1}{2} \sum\left(X_{i} Y_{j}-X_{j} Y_{i}\right)^{2}=|X|^{2}|Y|^{2}-\langle X, Y\rangle^{2}
$$

for vectors enables us to write

$$
\begin{aligned}
C(x) & =A(x)-B(x) \\
& =\int|x-c|^{\alpha-2} \rho(c)\left|\frac{x-c}{|x-c|} \wedge \int\right| x-\left.\left.a\right|^{\alpha-2}(x-a) \rho(a) d a\right|^{2} d c .
\end{aligned}
$$

Notice that $C(x) \geq 0$. Thus we have arrived at the representation formula

$$
\boldsymbol{\Delta}_{\infty} \mathbf{V}(\mathbf{x})=\alpha^{3} \mathbf{C}(\mathbf{x})+\alpha^{3}(\alpha-\mathbf{1}) \mathbf{B}(\mathbf{x}),
$$

which is particularly appealing for $\alpha=1$ and

$$
V(x)=\int|x-y| \rho(y) d y .
$$

Continuing the calculations, we find that

$$
\Delta V(x)=\alpha(\alpha-2+n) \int|x-c|^{\alpha-2} \rho(c) d c,
$$

and hence, after some simple manipulations

$$
|\nabla V|^{2} \Delta V=\alpha^{3}(\alpha-2+n) A .
$$

It follows that

$$
\begin{aligned}
|\nabla V|^{2} \Delta V & +(p-2) \Delta_{\infty} V \\
& =\alpha^{3}(n+\alpha+p-4) A(x)+\alpha^{3}(\alpha-2)(p-2) B(x) \\
& =\alpha^{3}[(2-\alpha)(p-2) C(x)+(n-p+\alpha(p-1)) B(x)] .
\end{aligned}
$$

In this formula we have command over the sign of

$$
|\nabla V|^{2} \Delta V+(p-2) \Delta_{\infty} V
$$

at least in the cases needed for the theorem. We may add that the logarithmic integral in the borderline case $p=n$ requires a separate, but similar, calculation leading to

$$
\begin{gathered}
\left|\nabla V_{0}\right|^{2} \Delta V_{0}+(n-2) \Delta_{\infty} V_{0} \\
=2(n-2) C(x)
\end{gathered}
$$

where $\alpha=0$ in the expression for $C(x)$. This concludes our proof of the Theorem. 
It is remarkable that the factor $n-p+\alpha(p-1)$ in front of $B(x)$ reveals the natural exponent $\alpha=(p-n) /(p-1)$; the term vanishes for this $\alpha$. Thus

$$
|\nabla \mathbf{V}|^{\mathbf{2}} \boldsymbol{\Delta} \mathbf{V}+(\mathbf{p}-\mathbf{2}) \boldsymbol{\Delta}_{\infty} \mathbf{V}=\alpha^{\mathbf{3}}(\mathbf{2}-\alpha)(\mathbf{p}-\mathbf{2}) \mathbf{C}(\mathbf{x})
$$

when $\alpha=(p-n) /(p-1)$, and $p>2$.

To this one may add that the method is rather flexible. For example, in the case of a variable exponent it works for

$$
\int|x-y|^{\alpha(y)} \rho(y) d y .
$$

One can also consider $V(x)+\langle a, x\rangle$ with an extra linear term.

\section{Riesz Potentials}

So far, we have assumed that the nonnegative density $\rho$ in the Riesz potential

$$
V(x)=\int|x-y|^{\alpha} \rho(y) d y
$$

is smooth. The restriction can easily be relieved because of the following theorem: the pointwise limit of an increasing sequence of $p$-superharmonic functions is either a $p$-superharmonic function or identically $+\infty$. Thus we immediately reach the case with lower semicontinuous $\rho$ 's. We point out that the discrete case

$$
\sum A_{j}\left|x-a_{j}\right|^{\alpha}
$$

follows if one regards the integrals as sums in disguise and takes into account a special reasoning concerning the poles $a_{j}$.

We can do more than that. Indeed, we can allow rather general measures.

Proposition. Let $\mu$ be a Radon measure on $\mathbb{R}^{n}$ satisfying the growth condition

$$
\int_{|y| \geq 1}|y|^{\alpha} d \mu(y)<\infty .
$$

The theorem holds for the Riesz potentials

$$
V(x)=\int|x-y|^{\alpha} d \mu(y)
$$

In other words, we have replaced $\rho(y) d y$ with $d \mu(y)$. The growth condition guarantees that $V(x)<\infty$ almost everywhere. In fact $V(x) \equiv \infty$ if $\int|y|^{\alpha} d \mu(y)=$ $+\infty$. See [P, Theorem 3.4, p. 78] about this.

Proof. Because of the increasing limit

$$
V(x)=\lim _{R \rightarrow \infty} \int_{|y|<R}|x-y|^{\alpha} d \mu(y)
$$

we may, in the proof, assume that the measure $\mu$ has compact support. To simplify the exposition, we confine ourselves to the case

$$
\alpha=\frac{p-n}{p-1}, \quad 2<p<n .
$$


The passage from integrals of the type $\int|x-y|^{\alpha} \rho(y) d y$ to the more general kind with the Radon measure is accomplished through a regularization, for example

$$
\rho_{t}(y)=\frac{1}{(4 \pi t)^{n / 2}} \int e^{-\frac{|y-\xi|^{2}}{4 t}} d \mu(\xi)
$$

where the heat kernel is present. We have

$$
\int \rho_{t}(y) d y=\int d \mu(\xi)=\mu\left(\mathbb{R}^{n}\right)=M .
$$

Let us denote

$$
V_{k}(x)=\int|x-y|^{\alpha} \rho_{t_{k}}(y) d y \quad(k=1,2,3, \cdots)
$$

where $t_{k} \longrightarrow 0+$ as $k \longrightarrow \infty$. According to the theorem each $V_{k}$ is $p$-superharmonic. It is not difficult to see that $V_{k} \longrightarrow V$ a.e., at least for a subsequence. The Proposition follows from the general theorem about a.e. convergence in [KM, Theorem 1.17], which assures that the limit fuction is $p$-superharmonic.

In the present situation a more direct proof is possible. It is based on a compactness argument in $W_{\text {loc }}^{1, p-1}\left(\mathbb{R}^{n}\right)$.

Alternative proof. A direct calculation yields

$$
\int_{B_{R}}\left|\nabla V_{k}\right|^{p-1} d x \leq 2\left(M \frac{n-p}{n-1}\right)^{p-1} \omega_{n-1} R, \quad k=1,2,3, \cdots .
$$

To obtain such a bound, free of $k$, we proceed as follows:

$$
\begin{aligned}
\left|\nabla V_{k}(x)\right| & \leq|\alpha| \int|x-y|^{\alpha-1} \rho_{k}(y) d y \\
\left|\nabla V_{k}(x)\right|^{p-1} & \leq|\alpha|^{p-1} \int|x-y|^{(\alpha-1)(p-1)} \rho_{k}(y) d y\left(\int \rho_{k}(y) d y\right)^{p-2} \\
& =|\alpha|^{p-1} M^{p-2} \int|x-y|^{1-n} \rho_{k}(y) d y \\
\int_{B_{R}}\left|\nabla V_{k}(x)\right|^{p-1} d x & \leq|\alpha|^{p-1} M^{p-2} \int \rho_{k}(y) \int_{B_{R}}|x-y|^{1-n} d x d y .
\end{aligned}
$$

The inner integral can be estimated as

$$
\int_{B_{R}}|x-y|^{1-n} d x \leq 2 R \omega_{n-1}
$$

since $y \in B_{R}$. This yields the desired bound.

According to the celebrated Banach-Saks theorem there exists a sequence of indices $k_{1}<k_{2}<\cdots$ such that for the arithmetic means

$$
W_{j}=\frac{V_{k_{1}}+V_{k_{2}}+\cdots+V_{k_{j}}}{j}
$$

we have that $\nabla W_{j} \longrightarrow \nabla V$ strongly in $L^{p-1}\left(B_{R}\right)$. Now we take advantage of the linear structure by concluding that each $W_{j}$ is a $p$-superharmonic function, because it can be written as a Riesz potential with the density $\left(\rho_{k_{1}}+\cdots+\rho_{k_{j}}\right) / j$. Hence

$$
\int\left\langle\left|\nabla W_{j}\right|^{p-2} \nabla W_{j}, \nabla \varphi\right\rangle d x \geq 0
$$


for each nonnegative test function $\varphi \in C_{0}^{\infty}\left(\mathbb{R}^{n}\right)$. Given $\varphi$, we take a ball $B_{R}$ containing its support. The strong convergence of the sequence $\left\{\nabla W_{j}\right\}$ in $L^{p-1}\left(B_{R}\right)$ and the elementary vector inequality

$$
\left.|| b\right|^{p-2} b-|a|^{p-2} a|\leq(p-1)| b-a \mid(|b|+|a|)^{p-2}, \quad p \geq 2,
$$

enable us to pass to the limit under the integral sign so that also

$$
\int\left\langle|\nabla V|^{p-2} \nabla V, \nabla \varphi\right\rangle d x \geq 0 \text {. }
$$

We could conclude that $V$ is a weak supersolution if we knew that $V$ belongs to $W_{\text {loc }}^{1, p}(\mathbb{R})$. Unfortunately, this is not always the case. For example, the fundamental solution is not in $W_{\text {loc }}^{1, p}\left(\mathbb{R}^{n}\right)$. A simple correction is required. Also the cut-off functions*

$$
W_{j}^{L}=\min \left\{W_{j}(x), L\right\}
$$

are weak $p$-supersolutions. The ordinary Caccioppoli estimate

$$
\int \zeta^{p}\left|\nabla W_{j}^{L}\right|^{p} d x \leq p^{p} L^{p} \int|\nabla \zeta|^{p} d x
$$

is available; cf. [L, Corollary 2.5]. By weak lower semicontinuity it also holds for

$$
V^{L}=\min \{V(x), L\} .
$$

Therefore, $\nabla V^{L} \in L_{\mathrm{loc}}^{p}\left(\mathbb{R}^{n}\right)$, so that $V^{L}$ is in the right Sobolev space.

As before, we can conclude that

$$
\int\left\langle\left|\nabla V^{L}\right|^{p-2} \nabla V^{L}, \nabla \varphi\right\rangle d x \geq 0,
$$

but this time it follows that $V^{L}$ is a weak $p$-supersolution. Then the increasing limit $V=\lim _{L \rightarrow \infty} V^{L}$ is $p$-superharmonic.

Strictly speaking, the conclusion is that the function

$$
\tilde{V}(x)=\operatorname{ess} \liminf _{y \rightarrow x} V(y)
$$

is $p$-superharmonic, because it is the increasing limit of the $p$-superharmonic functions ess liminf $V^{L}(y)$ as $L \longrightarrow \infty$. See [KM, Proposition 1.7]. The Lemma below concludes our proof.

Lemma (Brelot). At each point $x_{0}$ we have

$$
V\left(x_{0}\right)=\tilde{V}\left(x_{0}\right)=e s s \liminf _{x \rightarrow x_{0}} V(x),
$$

when $2-n<\alpha<0$.

For the sake of completeness we present the proof.

Proof. The function $|x-y|^{\alpha}$ is superharmonic and therefore we have

$$
f_{B\left(x_{0}, r\right)}|x-y|^{\alpha} d x \leq\left|x_{0}-y\right|^{\alpha}
$$

\footnotetext{
${ }^{*}$ This proof does not work if one cuts the original functions $V_{j}$ instead.
} 
for the volume average over the ball $B\left(x_{0}, r\right)$. It follows that

$$
\begin{aligned}
f_{B\left(x_{0}, r\right)} V(x) d x & =f_{B\left(x_{0}, r\right)} \int|x-y|^{\alpha} d \mu(y) d x \\
& =\int\left(f_{B\left(x_{0}, r\right)}|x-y|^{\alpha} d x\right) d \mu(y) \\
& \leq \int\left|x_{0}-y\right|^{\alpha} d \mu(y)=V\left(x_{0}\right) .
\end{aligned}
$$

This is merely a restatement of the fact that $V$ is a superharmonic function (in the ordinary sense).

It follows from Fatou's lemma that $V$ is lower semicontinuous. Hence

$$
\begin{aligned}
V\left(x_{0}\right) & \leq \liminf _{x \rightarrow x_{0}} V(x) \leq \operatorname{ess} \liminf _{x \rightarrow x_{0}} V(x) \\
& \leq \liminf _{r \rightarrow 0} f_{B\left(x_{0}, r\right)} V(x) d x \leq V\left(x_{0}\right)
\end{aligned}
$$

where the last inequality was proved above. Thus equality holds at each step.

\section{REFERENCES}

[CZ] M. CRANDALL \& J. ZHANG, Another way to say harmonic, Transactions of the American Mathematical Society 355, 2002, pp. 241-263. MR1928087 (2003k:35062)

[JLM] P. JUUTINEN, P. LINDQVIST \& J. MANFREDI, On the equivalence of viscosity solutions and weak solutions for a quasilinear equation, SIAM Journal on Mathematical Analysis 33, 2001, pp. 699-717. MR1871417 (2002m:35051)

[KM] T. KILPELÄINEN \& J. MALÝ, Degenerate elliptic equations with measure data and nonlinear potentials, Annali della Scuola Normale Superiore di Pisa, Cl. Sci. 4 19, 1992, pp. 591-613. MR1205885 (94c:35091)

[L] P. LINDQVIST, On the definition and properties of p-superharmonic functions, Journal für die Reine und Angewandte Mathematik 365, 1986, pp. 67-79. MR826152 (87e:31011)

[P] N. du PLESSIS, An Introduction to Potential Theory, Oliver \& Boyd, Edinburgh, 1970. MR0435422 (55:8382)

Department of Mathematics, Norwegian University of Science and Technology, NO7491 TrondheIM, NORWAY

E-mail address: lindqvist@math.ntnu.no

Department of Mathematics, University of Pittsburgh, Pittsburgh, Pennsylvania 15260

E-mail address: manfredi@pitt.edu 